\title{
Are 6-n-propylthiouracil (PROP) taster status and fungiform papillae (FP) density related to alcohol intake in a group of Irish adults?
}

\author{
S. O’Brien, E. Feeney, A. Scannell, A. Markey and E. R. Gibney \\ UCD Centre for Food \& Health, School of Agriculture, Food Science and Veterinary Medicine, University College Dublin, \\ Belfield, Dublin 4, Republic of Ireland
}

Phenylthiocarbamide and its chemically-related compound, 6- $n$-propylthiouracil (PROP), provide an extremely bitter taste to some individuals (super tasters; ST), a slightly bitter taste to some (medium tasters; MT) or no taste at all to others (non-tasters; NT) ${ }^{(1)}$. Studies suggest FP densities vary between taster groups, with ST having greater numbers of FP than NT on the anterior of their tongue ${ }^{(2)}$. ST are therefore more likely to experience greater bitter oral sensations from bitter-tasting foods and drinks, e.g. alcohol, compared with NT. Studies have also linked alcohol intake to PROP taster status ${ }^{(3)}$.

The present study aims to examine whether PROP taster status is related to alcohol intake in a group of Irish adults. The data were collected as part of a larger on-going project examining PROP taster status and its effect on fruit and vegetable intake. In the study ninetyfour adults (seventy-one females and twenty-three males) were recruited from the Dublin area. Alcohol intakes were assessed through diet histories and analysed using WISP ${ }^{\odot}$ (Tinuviel Software, Llanfechell, Anglesey, UK). PROP taster status was established using PROPimpregnated paper discs and general labelled magnitude scales (gLMS) as previously described ${ }^{(4)}$. Statistical analysis was carried out using SPSS (SPSS Inc., Chicago, IL, USA; ANOVA (post hoc) and correlation).

No significant differences were found for mean daily alcohol intake in adults when assessed using the PROP ratings from the gLMS, or when PROP ratings on the gLMS were converted to PROP-taster groups according to cut-off points previously described ${ }^{(5)}(P=0.091$ and $R^{2}-0.155$ respectively). No significant differences were observed for alcohol intake and FP density across the three groups $\left(R^{2} 0.0012\right)$.

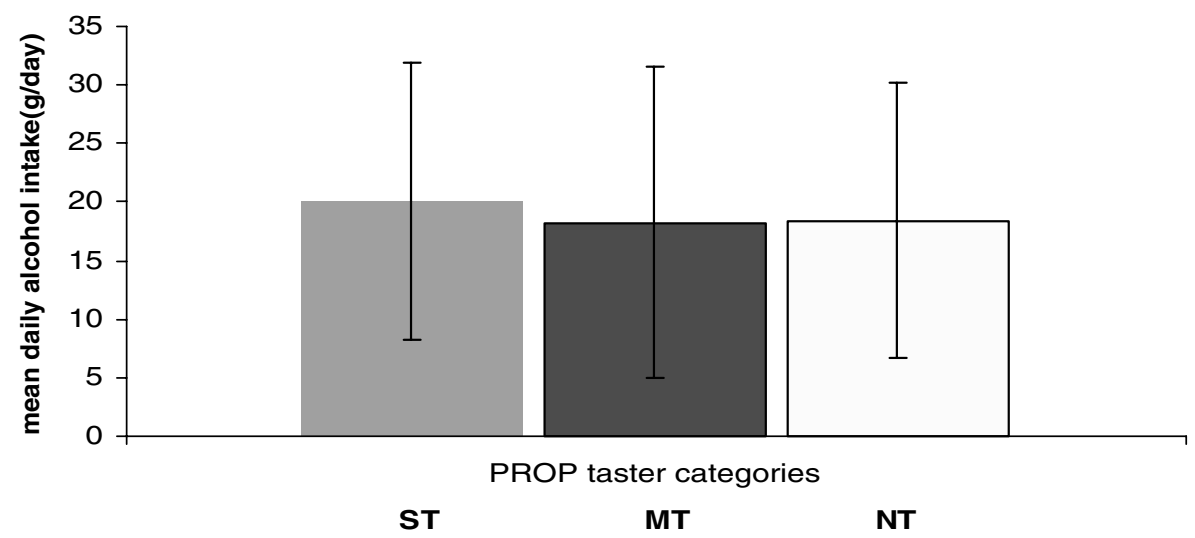

Figure. Values are means and standard deviations represented by vertical bars.

Mean daily alcohol intake was higher, although not significantly, in ST than NT. This finding is not in agreement with results from previous studies that suggest that ST experience heightened irritation from, and a lowered preference to, the bitter taste of wines and beers and therefore consume less alcoholic beverages ${ }^{(4)}$. However, it is known that alcohol intake is related to more than taste, and can also be associated with low socio-economic status, stress and many other factors ${ }^{(6)}$. An individual's ability to taste also diminishes with age; therefore, adults may not react in the same way to adverse taste ${ }^{(7)}$. Thus, PROP taster status and FP density are unsuitable predictors of alcohol intake.

This research is funded by Food Institutional Research Measures (FIRM), Department of Agriculture and Food, Dublin, Republic of Ireland.

1. Bartoshuk LM, Duffy VB \& Miller IJ (1994) Physiol Behav 56, 1165-1171.

2. Essicka GK, Chopra A, Guest S et al. (2003) Physiol Behav 80, 289-302.

3. Duffy VB, Davidson AC, Kidd JR et al. (2004) Alcohol Clin Exp Res 28, 1629-1637.

4. Fast K, Green BG \& Bartoshuk L (2002) Appetite 39, 75.

5. Zhao L, Kirkmeyer SV \& Tepper BJ (2003) Physiol Behav 78, 625-633.

6. Drommer M, Schrijvers CTM, Stronks K et al. (1999) Prev Med 29, 1-10.

7. Moiet J, Chris-Hazelhof E \& Heidema J (2003) Chem Senses 26, 845-860. 